\title{
A VLBI survey of compact broad absorption line quasars with balnicity index $\mathrm{BI}>0$
}

\author{
M. Kunert-Bajraszewska, M. Cegłowski, K. Katarzyński, and C. Roskowiński \\ Toruń Centre for Astronomy, Faculty of Physics, Astronomy and Informatics, NCU, Grudziadzka 5, 87-100 Toruń, Poland \\ e-mail: magda@astro.uni.torun.pl
}

Received 13 March 2015 / Accepted 11 May 2015

\section{ABSTRACT}

\begin{abstract}
Aims. Outflows manifest as broad absorption lines (BALs) in the quasars spectra. Although outflows are one of the most common astrophysical processes in the Universe, the BAL quasars are rare. Radio emission is another tool that can help to understand the phenomenon of BAL quasars. The aim of this paper is to study their orientation and age by very long baseline interferometry (VLBI) imaging and radio-loudness parameter distribution.

Methods. We performed high resolution radio observations of a new sample of ten BAL quasars using both the Very Long Baseline Array (VLBA) and the European VLBI Network (EVN) at $5 \mathrm{GHz}$. All the selected sources have balnicity indices (BI) more than 0 and radio flux densities less than $80 \mathrm{mJy}$ at $1.4 \mathrm{GHz}$. They are very compact with linear sizes of the order of a few tens of parsecs and radio luminosities at $1.4 \mathrm{GHz}$ above the FR I-FR II luminosity threshold.

Results. Most of the observed objects have been resolved at $5 \mathrm{GHz}$ showing one-sided, probably core-jet structures, typical for quasars. We discuss in detail their age and orientation based on the radio observations. We then used the largest available sample of BAL quasars to study the relationships between the radio and optical properties in these objects. We found that (1) the strongest absorption (high values of the balnicity index $\mathrm{BI}$ ) is connected with the lower values of the radio-loudness parameter, $\log R_{\mathrm{I}}<1.5$, and thus probably with large viewing angles; (2) the large span of the BI values in each bin of the radio-loudness parameter indicates that the orientation is only one of the factors influencing the measured absorption; (3) most of the radio-loud BAL quasars are compact, low luminosity objects with a wide range of jet power (although the highest values of BI seem to be associated with the lower values of jet power). In addition, we suggest that the short lifetime postulated for some compact active galactic nuclei could also explain the scarcity of the large-scale radio sources among BAL quasars.
\end{abstract}

Key words. galaxies: active - galaxies: evolution - quasars: absorption lines

\section{Introduction}

Broad absorption troughs of high (CIV $1549 \AA$ ) and low ionization resonant lines (Mg II $2800 \AA$ ) are seen in the spectra of both the radio-quiet and radio-loud quasars. Sources showing these features are called broad absorption line (BAL) quasars and their fraction among the whole quasar population varies from $15 \%$ to $26 \%$ depending on the definition used (Hewett \& Foltz 2003; Trump et al. 2006; Knigge et al. 2008; Gibson et al. 2009). BAL quasars (BALQSOs) are probably caused by the outflow of gas with high velocities and are part of the accretion process (Weyman et al. 1991). It is common to say that there are two models that could explain the presence of BALs in a small fraction of quasars, namely the orientation and evolution schemes. However, the growing number of observations of the BALQSOs indicate the complexity of BAL phenomenon and make the nature and origin of BALQSOs still an open issue. Thus, selecting one of the two mentioned models would be a simplification.

The spectroscopic and variability studies trying to characterize the UV absorber in BALQSOs generally indicate a complex behaviour of wind outflows. BALs in radio-quiet and radioloud quasars show wide range of depth changes from small to complete disappearance of troughs (Filiz et al. 2012). This can be caused, for example, by a change in the location of absorber along the line of sight (Capellupo et al. 2013). Recent observations of BAL variability in the sample of radio-loud BALQSOs supports the orientation dependence of the observed outflow (Welling et al. 2014).

Generally the orientation scenario implies that outflows are present in every quasar and the viewing angle of the source determines its detection. Thus, different wind geometries are proposed: near the equatorial plane (Murray et al. 1995), at mid inclination angles (Elvis 2000; Fine et al. 2011), or very close to the jet axis (Ghosh \& Punsly 2007; Reynolds et al. 2013). The radio variability study of some BALQSOs imply the existence of polar outflows from the inner regions of a thin disk (Zhou et al. 2006; Ghosh \& Punsly 2007). Nevertheless, objects with very high radio luminosities seem to be a minority among BALQSOs (Shankar et al. 2008).

On the other hand the VLA FIRST survey showed that most of the radio-loud BALQSOs are very compact (Becker et al. 2000). The high resolution VLBI observations of BALQSOs performed so far (Jiang \& Wang 2003; Kunert-Bajraszewska \& Marecki 2007; Kunert-Bajraszewska et al. 2010a; Liu et al. 2008; Montenegro-Montes et al. 2008; Gawroński \& KunertBajraszewska 2011; Bruni et al. 2012, 2013; Hayashi et al. 2013; Doi et al. 2009) have shown that many of them are unresolved even on a parsec scale. The analysis of the spectral shape, variability, and polarization properties of some of them (Montenegro-Montes et al. 2008; Kunert-Bajraszewska et al. 2010a; Liu et al. 2008) indicate that they are similar to 
Table 1. Radio-loud BAL quasar sample.

\begin{tabular}{|c|c|c|c|c|c|c|c|c|c|c|}
\hline $\begin{array}{c}\mathrm{RA}(\mathrm{J} 2000) \\
\mathrm{h} \mathrm{m} \mathrm{s} \\
(1)\end{array}$ & $\begin{array}{c}\operatorname{Dec}(\mathrm{J} 2000) \\
0, \prime \prime \\
(2) \\
\end{array}$ & $\begin{array}{l}z \\
(3)\end{array}$ & $\begin{array}{c}\mathrm{BI} \\
\left(\mathrm{km} \mathrm{s}^{-1}\right) \\
(4)\end{array}$ & $\begin{array}{c}S_{1.4 \mathrm{GHz}} \\
(\mathrm{mJy}) \\
(5)\end{array}$ & $\begin{array}{c}\log L_{1.4 \mathrm{GHz}} \\
\left(\mathrm{W} \mathrm{Hz}{ }^{-1}\right) \\
(6)\end{array}$ & $\begin{array}{c}S_{4.9 \mathrm{GHz}} \\
(\mathrm{mJy}) \\
(7)\end{array}$ & $\begin{array}{c}S_{8.4 \mathrm{GHz}} \\
(\mathrm{mJy}) \\
(8)\end{array}$ & $\begin{array}{l}\alpha_{\text {fit }} \\
(9) \\
\end{array}$ & $\begin{array}{c}\log R_{\mathrm{I}} \\
(10)\end{array}$ & $\begin{array}{c}\log P_{\text {jet }} \\
\left(\mathrm{W} \mathrm{Hz}^{-1}\right) \\
(11)\end{array}$ \\
\hline 081102.931 & 500724.52 & 1.84 & 371 & 24.9 & 26.8 & 10.9 & 11.9 & 0.41 & 2.1 & 38.7 \\
\hline 084224.395 & 063116.78 & 2.46 & 1974 & 51.0 & 27.4 & 25.6 & 21.2 & 0.49 & 2.8 & 39.3 \\
\hline 085641.566 & 424253.94 & 3.06 & 295 & 20.0 & 27.2 & 20.5 & 13.7 & 0.19 & 1.8 & 39.2 \\
\hline 104059.802 & 055524.78 & 2.44 & 4290 & 42.2 & 27.3 & 8.1 & 3.8 & 1.34 & 2.5 & 39.2 \\
\hline 133325.080 & 472935.36 & 2.62 & 152 & 44.7 & 27.4 & - & - & - & 2.1 & 39.3 \\
\hline 140126.163 & 520834.63 & 2.97 & 70 & 37.1 & 27.4 & - & - & - & 2.0 & 39.4 \\
\hline 145756.263 & 574446.90 & 2.13 & 17 & 78.9 & 27.4 & - & - & - & 2.7 & 39.3 \\
\hline 210757.683 & -062010.49 & 0.65 & 559 & 20.4 & 25.5 & - & - & - & 1.4 & 37.6 \\
\hline 223843.578 & 001648.05 & 3.47 & 13 & 36.9 & 27.6 & - & - & - & 2.2 & 39.6 \\
\hline 224800.717 & -090744.93 & 2.11 & 16 & 36.7 & 27.1 & - & - & - & 2.3 & 39.0 \\
\hline
\end{tabular}

Notes. Description of the columns: (1) and (2) source coordinates (J2000) extracted from FIRST; (3) redshift as measured from the SDSS; (4) balnicity index taken from Trump et al. (2006); (5) total flux density at $1.4 \mathrm{GHz}$ extracted from FIRST; (6) log of the radio luminosity at $1.4 \mathrm{GHz}$; (7) and (8) total flux density at $4.9 \mathrm{GHz}$ and $8.4 \mathrm{GHz}$ taken from DiPompeo et al. (2011); (9) spectral index using linear fit to all available radio data taken from DiPompeo et al. (2011); (10) radio-loudness, the radio-to-optical ( $i$-band) ratio of the quasar core (Kimball et al. 2011b), which were calculated from $z, S_{1.4 \mathrm{GHz}}, M_{\mathrm{i}}$ taken from Trump et al. (2006), and the assumption of a radio core spectral index of 0 and an optical spectral index of $-0.5,(11)$ jet power at $1.4 \mathrm{GHz}$ calculated using formula from Sikora et al. (2013; see also Sect. 3.3).

compact steep spectrum (CSS) objects and gigahertz peaked spectrum (GPS) objects, which are thought to be the progenitors of large-scale active galactic nuclei (AGNs; Fanti et al. 1995; O'Dea 1998). Compact BALQSOs are not oriented along a particular line of sight, although they are more often observed farther from the jet axis than are normal quasars (DiPompeo et al. 2011; Bruni et al. 2012). The alternative evolution scenario suggests then that every quasar has a BAL phase at the beginning of its lifetime (Becker et al. 2000; Gregg et al. 2006). The radio jets of the expanding source interact with the outflows and destroy the BALs before the source reaches the "adult" stage.

This paper is one of the two presenting high resolution radio observations and statistical analysis of compact radio-loud BALQSOs selected from the most recent available catalogues of BALQSOs created by Gibson et al. (2009) and Trump et al. (2006). The parent samples were selected from the Trump et al. (2006) catalogue and VLA FIRST survey (White et al. 1997) for further VLBI observations in two categories based on the values of absorption index (AI) and balnicity index (BI), which classify the source as a BALQSO. It should be noted here that the BAL definition is diffuse nowadays. The traditional BALs, quantified by the balnicity index $(\mathrm{BI})$, are defined as having $\mathrm{C}_{\mathrm{IV}}$ absorption troughs at least $2000 \mathrm{~km} \mathrm{~s}^{-1}$ wide (Weyman et al. 1991). However, this could potentially exclude the so-called mini-BALs with $\mathrm{BI}=0$. Therefore, the more liberal AI index, including quasars with weaker and much narrower absorption features (within $3000 \mathrm{~km} \mathrm{~s}^{-1}$ ) was introduced by Trump et al. (2006). The optical and radio analysis of BALQSOs from both groups $(\mathrm{AI}>0$ and $\mathrm{BI}=0$ and $\mathrm{BI}>0)$ revealed many differences between them indicating that they constitute two independent classes (Shankar et al. 2008; Knigge et al. 2008). However, if we assume that the absorption by outflows comes from the continuum of velocity widths (Ganguly et al. 2007) then the study of both classes of objects is important in order to understand the origin and nature of BALs.

In this paper we report high resolution radio observations of a sample of so-called traditional BALQSOs with balnicity index values $\mathrm{BI}>0$ and thus low radio fluxes. The results of the study of mini-BALQSOs $(\mathrm{AI}>0$ and $\mathrm{BI}=0)$ are presented in Cegłowski et al. (2015).

\section{Sample selection}

\subsection{Radio observations}

In order to build a sample of compact radio-loud BALQSOs we matched the optical positions of BALQSOs from the Trump et al. (2006) catalogue to FIRST coordinates (White et al. 1997) in a radius of $10 \mathrm{arcsec}$. In practice it appeared that for most of the objects $(\sim 96 \%)$ the separation between FIRST and SDSS position is below 2 arcsec. We also limited our sample to sources with integrated flux densities $S_{1.4 \mathrm{GHz}}>2 \mathrm{mJy}$ and side lobe probabilities less than 0.1 . In the next step we excluded from this initial sample objects with additional radio counterparts within 60 arcsec of the SDSS position. These extended sources constituted $\sim 12 \%$ of the initial sample. This approach allowed us to avoid doubts about all the components belonging to a single object and the identification of the core. Finally, our sample consisted of 309 quasars classified as BALQSOs according to the extended absorption index (AI) definition proposed by Trump et al. (2006), which includes BAL absorption features at lower outflow velocities (within $3000 \mathrm{~km} \mathrm{~s}^{-1}$ ). The traditional BALs are defined as having $\mathrm{C}_{\mathrm{IV}}$ absorption troughs at least $2000 \mathrm{~km} \mathrm{~s}^{-1}$ wide and at least $10 \%$ below the continuum at maximum depth (Weyman et al. 1991) and are quantified by the balnicity index (BI). Trump et al. (2006) calculate values of both the AI and BI indices for all quasars. Therefore, our sample in a natural way falls into two groups: 204 objects with $\mathrm{AI}>0$ and $\mathrm{BI}=0$ (AI sample) and 105 objects with $\mathrm{AI}>0$ and $\mathrm{BI}>0$, the so called "true BALQSOs" (BI sample).

We have selected 31 sources with the largest $1.4 \mathrm{GHz}$ flux densities from both groups for further high resolution VLBI observations. Since statistically the BI sources are weaker than AI quasars, this selection criterion means that the AI group ( $\mathrm{AI}>0$, $\mathrm{BI}=0$ ) consists of the 16 bright sources with flux densities $S_{1.4 \mathrm{GHz}}>150 \mathrm{mJy}$. The second $\mathrm{BI}$ group (AI $>0, \mathrm{BI}>0$ ) consists of 15 much weaker objects with flux densities in the range $20-80 \mathrm{mJy}$ at $1.4 \mathrm{GHz}$. However, five of the weak sources from the BI sample were already included in the sample of Montenegro-Montes et al. (2008) and thus were not observed by us. In this paper we report high resolution $5 \mathrm{GHz}$ observations of ten quasars from the BI group, which are presented in Table 1. 
All the $C$-band observations were made in February 2013 (EVN and VLBA) and December 2013 (VLBA) in snapshot mode with phase referencing. Each target source together with its associated phase reference source was observed for $\sim 60 \mathrm{~min}$ including telescope drive times. In the case of VLBA observations the correlation was performed with the new Distributed FX (DiFX) software correlator (Deller et al. 2011) at the National Radio Astronomy Observatory (NRAO) in Socorro. The European VLBI Network (EVN) observations were made using the antennas in Jodrell Bank, Westerbork, Effelsberg, Onsala, Medicina, Torun, Shanghai, Noto, Yebes, and Hartebeesthoek. The data were correlated at the Joint Institute for VLBI in Europe (JIVE) correlator in Dwingeloo.

Data reduction (including editing, amplitude calibration, instrumental phase corrections, and fringe-fitting) was performed with the standard procedure using the NRAO AIPS ${ }^{1}$ software. After this stage the calibrated data were imported into Difmap package (Shepherd et al. 1997) to produce the final Stokes $I$ images and fit the sources with a number of discrete circular Gaussian components. The fitting was done directly on the final, self-calibrated visibility data using the MODELFIT programme. The final images of the radio-loud BALQSOs are presented in Fig. 1 and the modelfit parameters are listed in Table 2. The unresolved sources were classified as "Single" (S) and for the others we proposed "Core-Jet" (CJ) classification. We note here that we call this classification "suggested" because it is based only on observations made at one frequency. In the case of resolved structure the brightest component is referred to as $\mathrm{C} 1$.

\subsection{Statistical studies}

The statistical analysis of the properties of compact radio-loud BALQSOs presented in this paper was made using the most recent available catalogue of BALQSOs created by Gibson et al. (2009) which is drawn from the Sloan Digital Sky Survey (SDSS) data release 5 instead of the Trump et al. (2006) catalogue based on the SDSS/DR3. We then matched the optical positions from the Gibson et al. (2009) catalogue to the FIRST radio positions with the same selection criteria as described in Sect. 2.1 and found 303 radio-loud BALQSOs of which again $\sim 12 \%$ are extended objects. Thus, the number of compact sources is 267 . It should be noted here that Gibson et al. (2009) identify the BALQSOs based on the "traditional" balnicity index BI (Weyman et al. 1991) and a modified $\mathrm{BI}_{0}$ which integrates absorption starting from $0 \mathrm{~km} \mathrm{~s}^{-1}$ instead of the traditional $3000 \mathrm{~km} \mathrm{~s}^{-1}$ in the lines of $\mathrm{C}_{\mathrm{IV}}, \mathrm{Si}_{\mathrm{IV}}, \mathrm{Al}_{\mathrm{III}}$, and $\mathrm{Mg}_{\mathrm{II}}$. The group of 267 compact radio-loud BALQSOs contains objects with $\mathrm{BI}_{0}>0$ in at least one of these lines.

We note here that the different methods used by Gibson et al. (2009) and Trump et al. (2006) in classifying objects as BALQSOs cause some differences between these two catalogues. Two out of ten sources $(1333+4729$ and $1457+5744)$ selected from Trump et al. (2006) and observed by us in the VLBI technique are not included in the Gibson et al. (2009) catalogue.

Finally, we note again that we removed from the sample studied here about $12 \%$ of the radio-loud BALQSOs with more than one radio counterpart in the radius of 60 arcsec. We argue, that this operation did not introduce any additional selection effects to the obtained results. The calculated $\mathrm{BI}_{0}$ for most of the large-scale objects is much less than 3000 and thus their removal did not affect the discussed conclusions, e.g. BI vs. $\log R_{\mathrm{I}}$ relation or BI vs. jet power plot (see Figs. 2 and 4).

\footnotetext{
http://www . aips.nrao.edu
}

Throughout the paper, we assume a cosmology with $H_{0}=$ $70 \mathrm{~km} \mathrm{~s}^{-1} \mathrm{Mpc}^{-1}, \Omega_{\mathrm{M}}=0.3, \Omega_{\Lambda}=0.7$. The adopted convention for the spectral index definition is $S \propto v^{-\alpha}$.

\section{Results and discussion}

\subsection{Characteristics of the VLBI sample}

We have made high resolution $5 \mathrm{GHz}$ EVN/VLBA observations of ten radio-loud BALQSOs with balnicity index $\mathrm{BI}>0$ and flux densities $S_{1.4 \mathrm{GHz}}>20 \mathrm{mJy}$. We detected all the objects, but three of them $(0856+4242,2107-0620,2238+0016)$ still remain unresolved at $5 \mathrm{GHz}$. The resolved ones show one-sided morphology probably indicating the core-jet type of structure (Fig. 1), typical for quasars. The small linear sizes $(\ll 1 \mathrm{kpc})$ of the observed quasars and their high luminosity (Table 2) may indicate the young age of these AGNs. However, lack of observations at other radio frequencies of most of the sources prevented us from analysing their spectra. Four of our objects have been observed with EVLA at 4.9 and $8.4 \mathrm{GHz}$ (DiPompeo et al. 2011) and two sources have X-ray flux estimations (Wang et al. 2008). Simple linear models have been fitted to the spectra of four our sources by DiPompeo et al. (2011) revealing a flat spectral index $(\alpha<0.5)$ in the cases of $0811+5007$ and $0856+4242$, and a steep spectral index for resolved sources $0842+0631$ and $1040+0555$. The evolutionary status of the compact BALQSOs have already been discussed by a few authors (Kunert-Bajraszewska \& Marecki 2007; Montenegro-Montes et al. 2008; Kunert-Bajraszewska et al. 2010a; Bruni et al. 2013), who suggest that they belong to the class of young AGNs, namely the compact steep spectrum (CSS) sources (see also the discussion in Sect. 3.3).

Using the high resolution VLBI observations of our quasars we have calculated the brightness temperatures (Eq. (A.4) in the Appendix) of their central components as indicated in the images and in Table 2. The calculated values of $T_{\mathrm{b}}(\mathrm{VLBI})$ are in the range $10^{8}-10^{10}$, although these values should be treated as the lower limits since we are restricted by the resolution of our observations. The brightness temperatures $T_{\mathrm{b}, 1.4 \mathrm{GHz}} \lesssim 10^{5} \mathrm{~K}$ and radio luminosity $L_{1.4 \mathrm{GHz}} \lesssim 10^{24} \mathrm{~W} \mathrm{~Hz}^{-1}$ are thought to be associated with stellar components of luminous starburts (Condon 1992; Condon et al. 2013). Therefore, the radio luminosities and values of the brightness temperatures of our BALQSOs indicate the AGN origin of their radio emission. On the other hand their values of the brightness temperatures do not exceed the inverse Compton brightness temperature limit of $10^{12} \mathrm{~K}$ and the empirical value of $10^{11} \mathrm{~K}$ (Lähteenmäki et al. 1999) that could indicate the emission boosting. However, three sources from our sample were previously reported in the literature as polar BALQSOs (Zhou et al. 2006; Ghosh \& Punsly 2007), which means objects with lines of sight close to the radio-jet axis. This classification was made based on the high values of the variability brightness temperature $T_{\mathrm{b}}$ (var) of these quasars exceeding the theoretical limit of $10^{12} \mathrm{~K}$. We discuss this issue in the next paragraph.

\subsection{Statistics on the parent sample}

\subsubsection{Core radio-to-optical ratios}

Following Kimball et al. (2011b) we have calculated the radioloudness parameter $R_{\mathrm{I}}$ defined as the radio-to-optical ( $i$-band) ratio of the quasar core for all the BALQSOs selected by us from the most recent catalogue created by Gibson et al. (2009). 

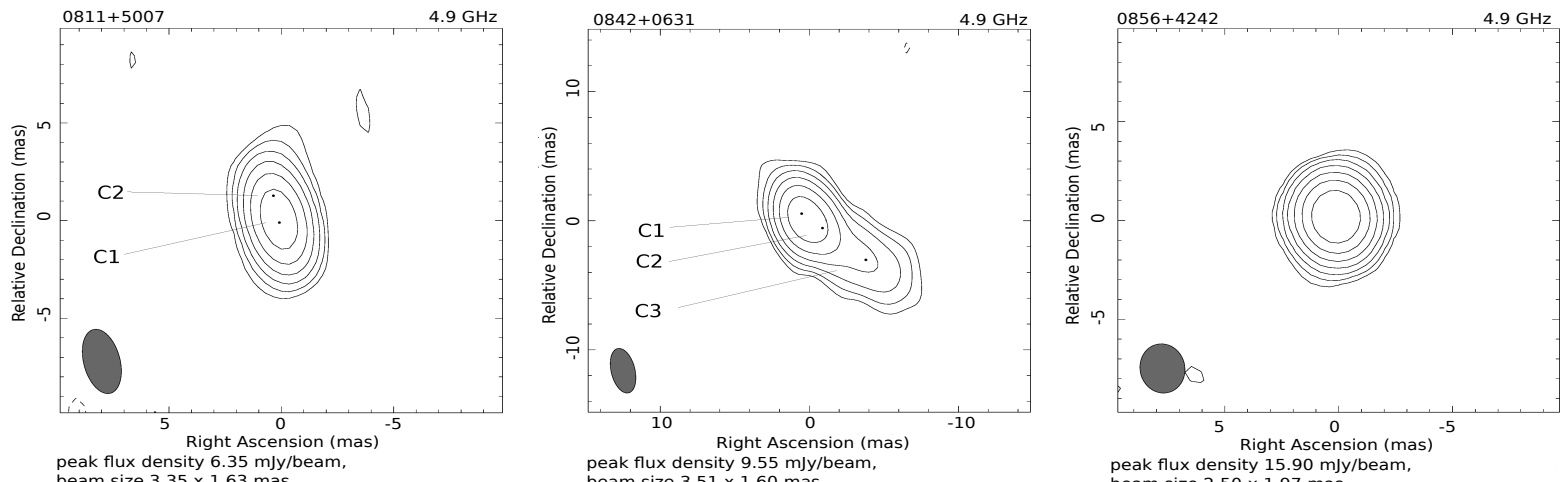

peak flux density $6.35 \mathrm{mJy} / \mathrm{be}$
beam size $3.35 \times 1.63 \mathrm{mas}$

first contour level $0.12 \mathrm{mJy} / \mathrm{beam}$

peak flux density $9.55 \mathrm{mJy} / \mathrm{beam}$,

first contour level $0.17 \mathrm{mJy} / \mathrm{beam}$
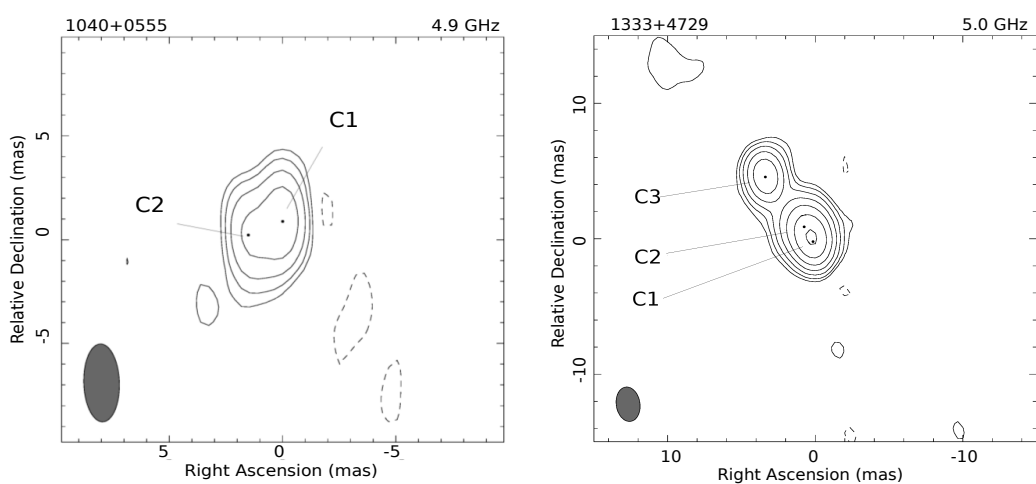

peak flux density $1.58 \mathrm{mJy} / \mathrm{beam}$

beam size $3.85 \times 1.58 \mathrm{mas}$,
first contour level $0.11 \mathrm{mJy} / \mathrm{beam}$

Right Ascension (mas)
peak flux density $7.17 \mathrm{mJy} / \mathrm{beam}$,
beam size $2.56 \times 1.61 \mathrm{mas}$,

first contour level $0.10 \mathrm{mJy} / \mathrm{beam}$

peak flux density $15.90 \mathrm{mJy} / \mathrm{beam}$

first contour level $0.12 \mathrm{mly} / \mathrm{beam}$
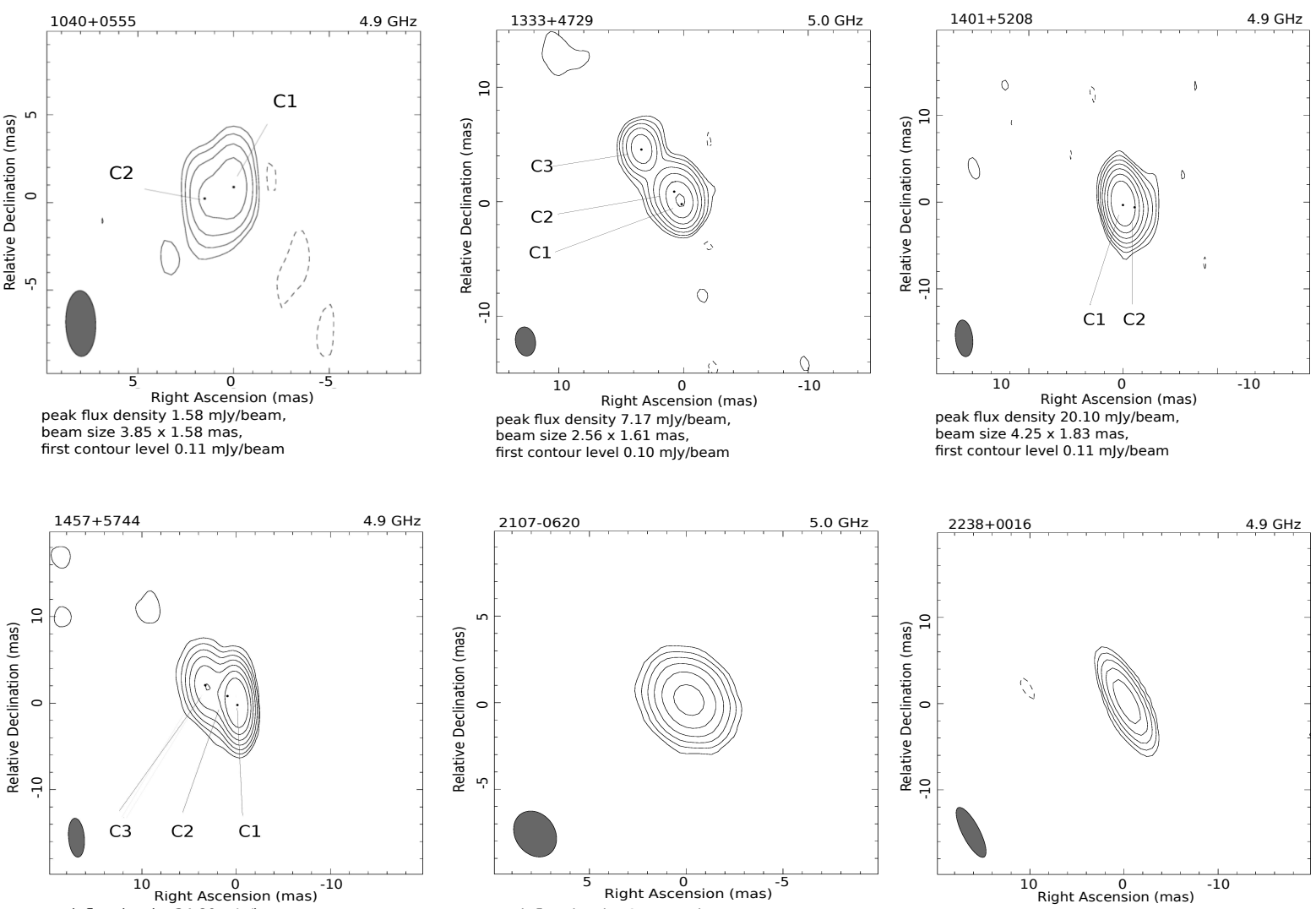

peak flux density $34.80 \mathrm{~mJ} / \mathrm{b}$ /bam,

beam size $4.52 \times 1.69 \mathrm{mas}$,

peak flux density $6.75 \mathrm{mJy} /$ beam

beam size $2.76 \times 2.10$ mas,

peak flux density $2.48 \mathrm{mJy} / \mathrm{beam}$,

beam size $6.38 \times 1.85$ mas,

(evel $0.09 \mathrm{~mJ} / \mathrm{beam}$

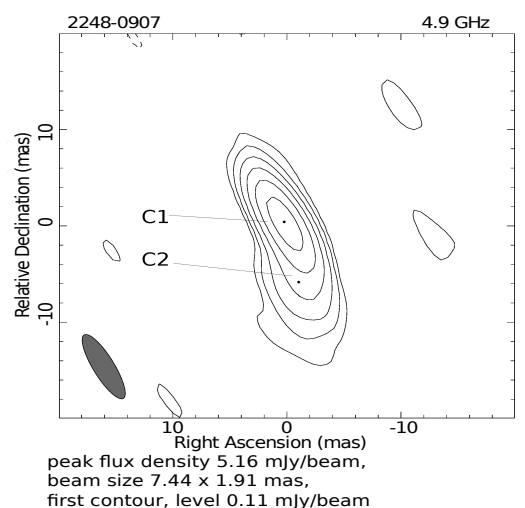

Fig. 1. Ten BALQSOs at $5 \mathrm{GHz}$. Two were made with EVN $(1333+4729,2107-0620)$ and the others with VLBA. Contours increase by a factor of 2 , and the first contour level corresponds to $\approx 3 \sigma$. 
Table 2. Results of VLBA and EVN observations.

\begin{tabular}{|c|c|c|c|c|c|c|c|c|}
\hline $\begin{array}{c}\mathrm{RA}(\mathrm{J} 2000) \\
\text { h m s } \\
(1)\end{array}$ & $\begin{array}{c}\operatorname{Dec}(\mathrm{J} 2000) \\
\circ, \prime \prime \\
(2) \\
\end{array}$ & $\begin{array}{c}\text { Comp. } \\
(3) \\
\end{array}$ & $\begin{array}{c}S_{5 \mathrm{GHz}} \\
(\mathrm{mJy}) \\
(4) \\
\end{array}$ & $\begin{array}{c}\theta \\
(\mathrm{mas}) \\
(5)\end{array}$ & $\begin{array}{c}\text { LAS } \\
\text { (mas) } \\
(6) \\
\end{array}$ & $\begin{array}{l}\text { LLS } \\
(\mathrm{pc}) \\
(7) \\
\end{array}$ & $\begin{array}{c}T_{\mathrm{b}}(\mathrm{VLBI}) \\
\left(10^{9} \delta^{-1} \mathrm{~K}\right) \\
(8) \\
\end{array}$ & $\begin{array}{l}\text { Type } \\
(9) \\
\end{array}$ \\
\hline 081102.931 & 500724.52 & $\begin{array}{l}\mathrm{C} 1 \\
\mathrm{C} 2\end{array}$ & $\begin{array}{l}6.4 \\
0.8\end{array}$ & $\begin{array}{l}0.7 \\
0.6\end{array}$ & 1.5 & 12.6 & 2.9 & CJ \\
\hline 084224.395 & 063116.78 & $\begin{array}{l}\text { C1 } \\
\text { C2 } \\
\text { C } 3\end{array}$ & $\begin{array}{c}11.5 \\
6.2 \\
4.4\end{array}$ & $\begin{array}{l}1.5 \\
1.4 \\
2.9\end{array}$ & 6.8 & 55.1 & 1.3 & $\mathrm{CJ}$ \\
\hline 085641.566 & 424253.94 & $\mathrm{C}$ & 17.2 & 0.5 & 0.5 & 3.8 & 18.2 & $\mathrm{~S}$ \\
\hline 104059.802 & 055524.78 & $\begin{array}{l}\mathrm{C} 1 \\
\mathrm{C} 2\end{array}$ & $\begin{array}{l}1.8 \\
0.7\end{array}$ & $\begin{array}{l}0.8 \\
0.1\end{array}$ & 2.1 & 17.0 & 0.7 & CJ \\
\hline 133325.080 & 472935.36 & $\begin{array}{l}\mathrm{C} 1 \\
\mathrm{C} 2 \\
\mathrm{C} 3\end{array}$ & $\begin{array}{l}5.7 \\
5.1 \\
3.3\end{array}$ & $\begin{array}{l}0.6 \\
1.7 \\
0.5\end{array}$ & 6.0 & 47.9 & 4.0 & $\mathrm{CJ}$ \\
\hline 140126.163 & 520834.63 & $\begin{array}{l}\mathrm{C} 1 \\
\mathrm{C} 2\end{array}$ & $\begin{array}{l}21.4 \\
2.4\end{array}$ & $\begin{array}{l}0.8 \\
1.9\end{array}$ & 1.2 & 9.3 & 9.6 & $\mathrm{CJ}$ \\
\hline 145756.263 & 574446.90 & $\begin{array}{l}\text { C1 } \\
\text { C2 } \\
\text { C } 3\end{array}$ & $\begin{array}{c}33.5 \\
7.8 \\
7.2\end{array}$ & $\begin{array}{l}0.4 \\
1.6 \\
1.9\end{array}$ & 4.2 & 34.9 & 40.1 & $\mathrm{CJ}$ \\
\hline $\begin{array}{l}210757.683 \\
223843.578\end{array}$ & $\begin{array}{c}-062010.49 \\
001648.05\end{array}$ & $\begin{array}{l}\mathrm{C} \\
\mathrm{C}\end{array}$ & $\begin{array}{l}6.7 \\
2.9\end{array}$ & $\begin{array}{l}0.4 \\
1.2\end{array}$ & $\begin{array}{l}0.4 \\
1.2\end{array}$ & $\begin{array}{l}2.8 \\
8.8\end{array}$ & $\begin{array}{l}4.8 \\
0.6\end{array}$ & $\begin{array}{l}S \\
S\end{array}$ \\
\hline 224800.717 & -090744.93 & $\begin{array}{l}\mathrm{C} 1 \\
\mathrm{C} 2\end{array}$ & $\begin{array}{l}6.6 \\
2.0\end{array}$ & $\begin{array}{l}1.5 \\
3.1\end{array}$ & 6.0 & 49.9 & 0.6 & $\mathrm{CJ}$ \\
\hline
\end{tabular}

Notes. Description of the columns: (1) and (2) source coordinates (J2000) extracted from FIRST; (3) components as indicated on the images; (4) flux density measured with the VLBA or EVN; (5) deconvolved major axis of the Gaussian fit; (6) largest angular size (LAS) measured at $5 \mathrm{GHz}$ image, LAS is defined as a separation between the two outermost Gaussian components or the size of the deconvolved component major axis (in the case of unresolved objects); (7) largest linear size (LLS) calculated based on the LAS; (8) brightness temperature calculated using Eq. (A.4); (9) suggested type of radio morphology.

Since all these sources are unresolved on FIRST resolution we assumed that their integrated $1.4 \mathrm{GHz}$ flux corresponds to a core flux and adopted the radio core spectral index and optical spectral index with a value of 0 and -0.5 , respectively. The histogram in Fig. 2 shows the number of BALQSOs versus the radio-loudness parameter $R_{\mathrm{I}}$. The peak of this distribution is in the range $1<\log R_{\mathrm{I}}<1.5$. Most of the quasars from the VLBI sample have values of $\log R_{\mathrm{I}}>2$ (Table 1 ) so they belong to the tail of $\log R_{\mathrm{I}}$ distribution for BALQSOs. It has been suggested that the radio-to-optical ratio of the quasar core is a better statistical measure of core boosting and therefore of orientation than the previously used core-to-lobe $(R)$ ratio (Wills \& Brotherton 1995). Recently Kimball et al. (2011b) showed that both parameters, $R_{\mathrm{I}}$ and $R$, are correlated which supports the hypothesis that they are indicative of the line-of-sight orientation. Objects with $\log R_{\mathrm{I}}>2.5$ are probably viewed close to the radio jet axis and quasars with $\log R_{\mathrm{I}}<1.5$ are thought to be those with large viewing angles. The radio-loudness distribution of BALQSOs can indicate that most of them are seen close to the disk plane.

The core-jet structures of quasars from the VLBI sample corresponds to the higher values of the radio-loudness parameter, $\log R_{\mathrm{I}}>2$. Our selection criteria caused that they constitute the "most extreme" cases among the BALQSOs. The one exception is the unresolved source 2107-0620. The low value of $\log R_{\mathrm{I}}$ and its X-ray weakness (Wang et al. 2008) may imply that this source is viewed close to the plane of the accretion disk.

\subsubsection{Variability brightness temperatures and viewing angle determination}

The maximum brightness temperature of powerful extragalactic radio sources is limited to the value of $10^{12} \mathrm{~K}$ by the inverse
Compton catastrophe. Using the total flux density variations of a sample of extragalactic radio sources Lähteenmäki et al. (1999) showed that the individual values of the intrinsic brightness temperature clustering around the equipartition limit of $10^{11} \mathrm{~K}$, in accordance with Readhead (1994). In the case of Doppler boosted sources $(\delta>1)$ the variability brightness temperatures are much larger than the VLBI brightness temperatures.

We looked for variable radio-loud BALQSOs in the parent sample selected from Gibson et al. (2009) using the method described by Zhou et al. (2006). We compared the FIRST and the NRAO VLA Sky Survey (NVSS; Condon et al. 1998) fluxes of these quasars in order to find differences implying the radio variability. The NVSS and FIRST surveys were carried out at the same frequency covering the same sky area but with different flux limit of the sources, different resolution, and at different times. We searched for counterparts of the selected quasars in the NVSS within $10^{\prime \prime}$ matching radius.

To classify the radio-loud BALQSO as a variable source we computed the variability ratio (VR) and the significance of the radio flux variability $\left(\sigma_{\mathrm{var}}\right)$ for each quasar as proposed by Zhou et al. (2006). The variability ratio (VR) is defined as

$\mathrm{VR}=\frac{F_{\text {peak }}}{N_{\text {int }}}$,

where $F_{\text {peak }}$ is the FIRST peak flux density and $N_{\text {int }}$ is the NVSS integrated flux density, and the significance of the radio flux variability $\left(\sigma_{\mathrm{var}}\right)$ is

$\sigma_{\text {var }}=\frac{F_{\text {peak }}-N_{\text {int }}}{\sqrt{\sigma_{F_{\text {peak }}}^{2}+\sigma_{N_{\text {int }}}^{2}}}$,

where the $\sigma_{F_{\text {peak }}}$ and $\sigma_{N_{\text {int }}}$ are the uncertainties of the FIRST peak flux densities and NVSS integrated flux densities, respectively. 


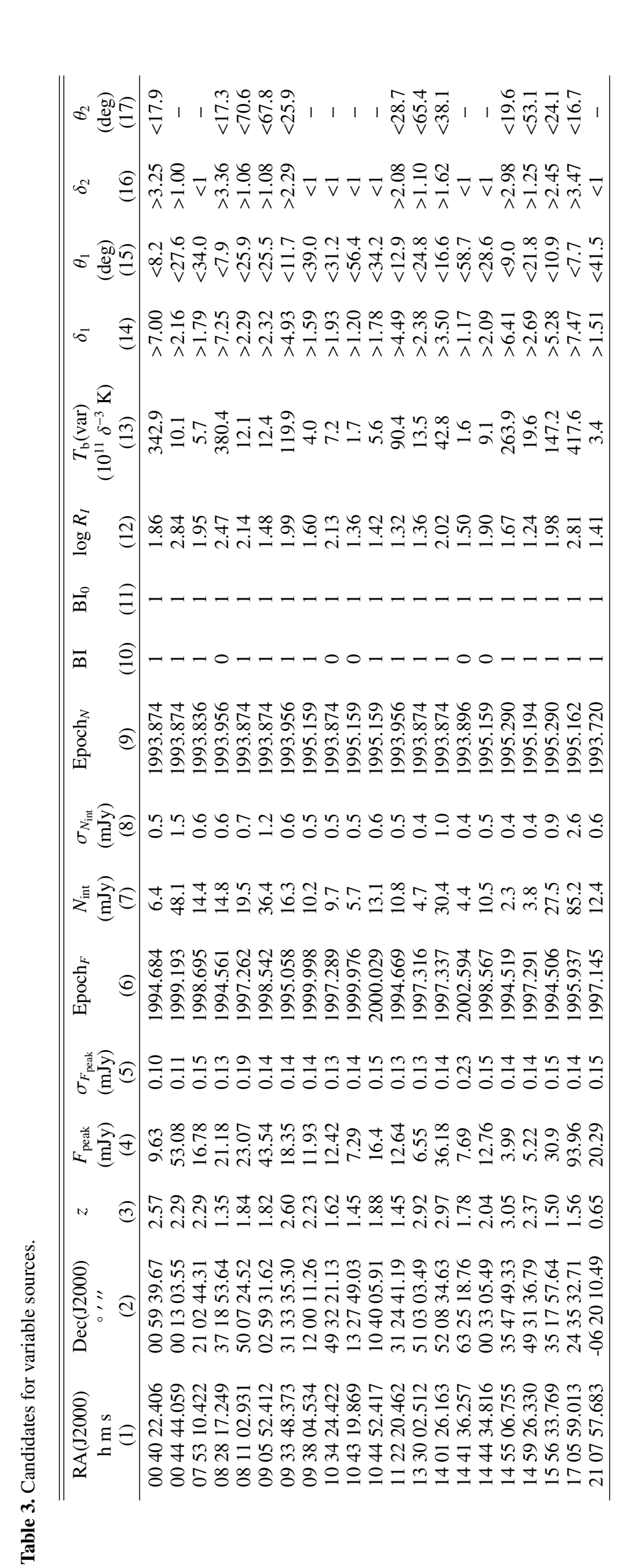

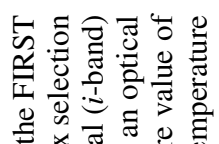

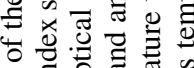

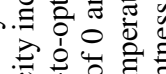

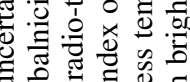

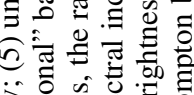
商:0 ฮี

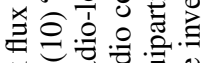

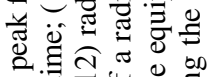

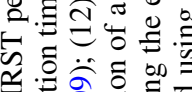

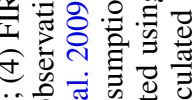
ñ 令 金氜芯宁

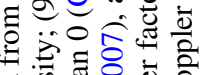

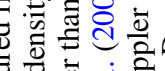

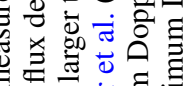
邑.

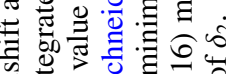

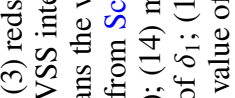

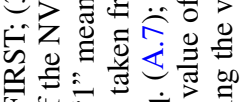

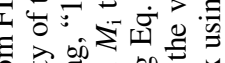

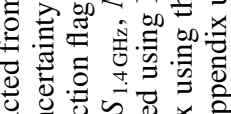

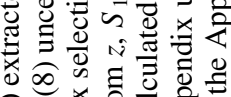

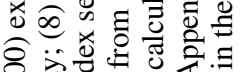

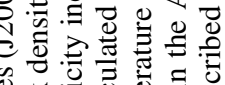

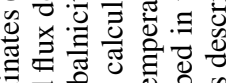

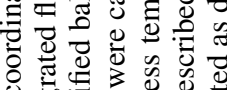

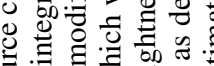

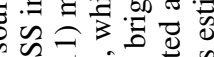

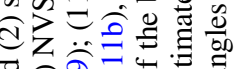
증ㅎㅇㅁㅇㅇㅎㅇ

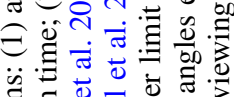

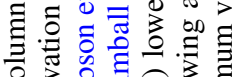

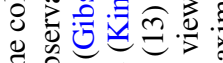

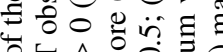

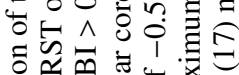

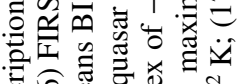

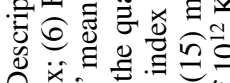

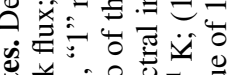

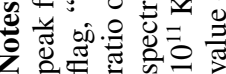


M. Kunert-Bajraszewska et al.: A VLBI survey of compact broad absorption line quasars with balnicity index BI $>0$
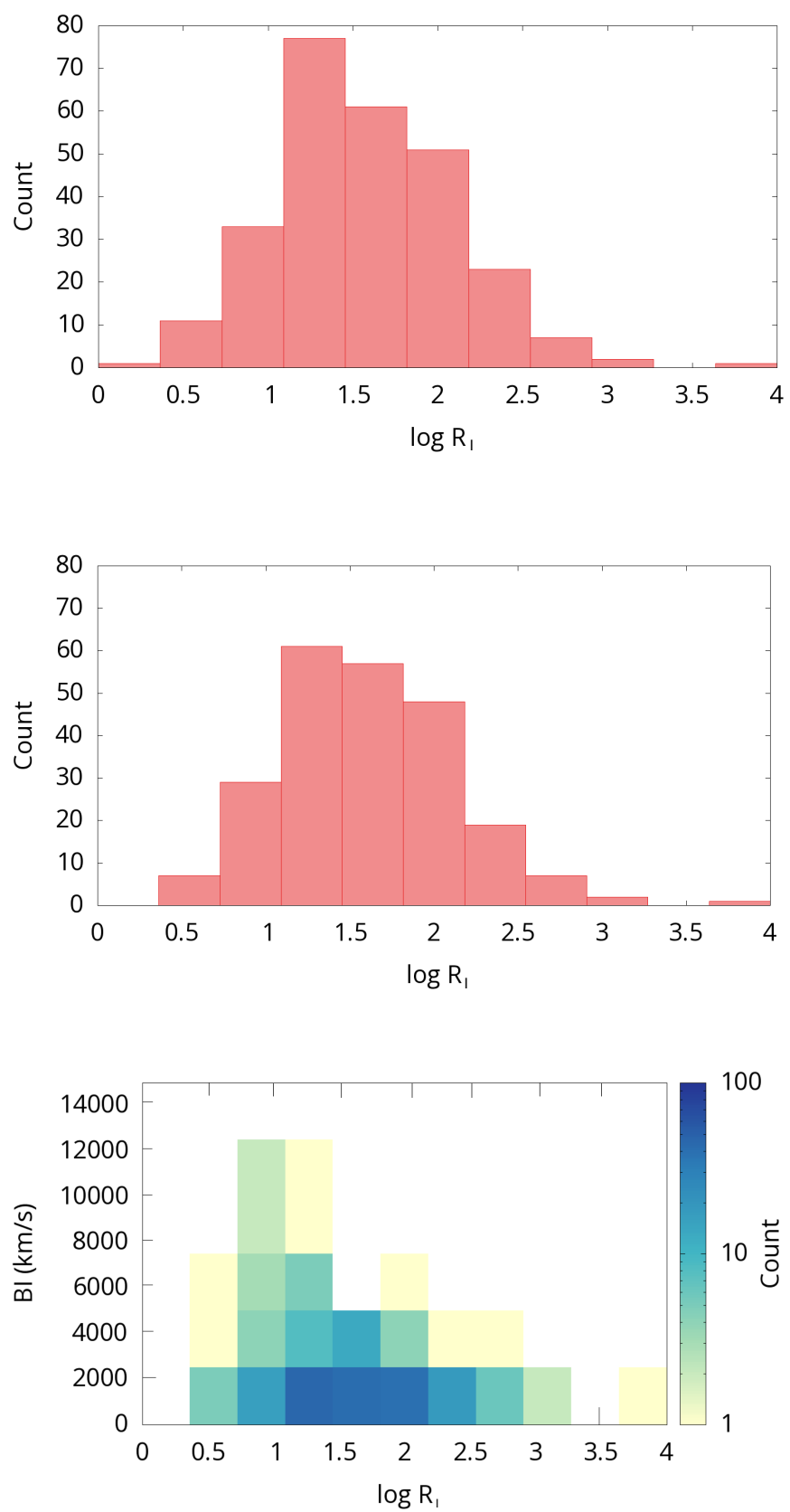

Fig. 2. Top: distribution of the radio-loudness parameter $R_{\mathrm{I}}$ (Kimball et al. 2011b) for the whole sample of radio-loud BALQSOs selected from Gibson et al. (2009) as described in Sect. 2. Middle: the same histogram but limited to the sources with $\mathrm{BI}_{0}\left(\mathrm{C}_{\mathrm{IV}}\right)>0$. Bottom: radioloudness vs. the modified balnicity index $\mathrm{BI}_{0}\left(\mathrm{C}_{\mathrm{IV}}\right)$ for the same sample of radio-loud BALQSOs.

BALQSOs with VR $>1$ and $\sigma_{\text {var }}>3$ were selected as candidates for variable sources. Finally, the sample consists of 21 candidates for variable BALQSOs and is presented in Table 3.

Using Eq. (A.7) we have calculated variability brightness temperature for all these objects. We then estimated the viewing angle $\theta$ of each object, defined as an angle between the jet axis and the observer, as the maximum of the function (Ghosh \& Punsly 2007)

$\cos \theta \leqslant \max \left[\frac{\delta-\sqrt{1-\beta^{2}}}{\delta \beta}\right]$,

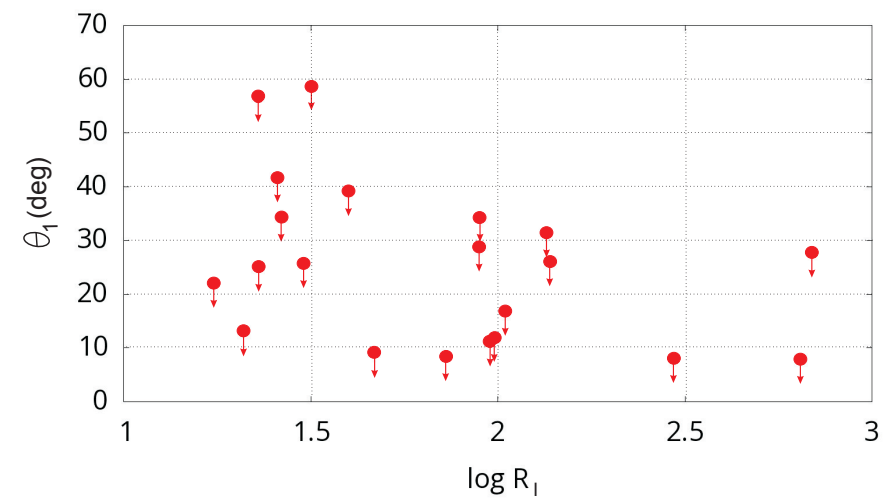

Fig. 3. Upper limits of the viewing angles $\left(\theta_{1}\right)$ vs. radio-loudness parameter $R_{\mathrm{I}}$ for the sources from Table 3 .

where $\beta$ is the velocity of the jet and $\delta$ is the Doppler factor. As was discussed at the beginning of this section, the intrinsic brightness temperature of extragalactic radio sources is probably in the range of $10^{11}-10^{12} \mathrm{~K}$ and this allows us to estimate the minimum Doppler factor that avoids the inverse Compton catastrophe to be $\delta_{1}=\left(\frac{T_{\mathrm{b}}(\mathrm{var})}{10^{11} K}\right)^{1 / 3}$ and $\delta_{2}=\left(\frac{T_{\mathrm{b}}(\mathrm{var})}{10^{12} \mathrm{~K}}\right)^{1 / 3}$, respectively. We then looked for the maximum value of function 3 for both $\delta_{1}$ and $\delta_{2}$ in order to determine the range where the maximum viewing angle of the quasar should be found (see Appendix for details).

As a result we have obtained a wide range of viewing angles reaching the value $\gtrsim 45^{\circ}$ in a few cases. The most populated subgroup of BALQSOs with $\log R_{\mathrm{I}}<1.5$ have the maximum viewing angle in the range 59-68 which constitutes the lower limit. This means that the viewing angle of the radio weakest BALQSOs is in the range $0-68^{\circ}$ (see also Fig. 3).

Three of the BALQSOs from the VLBI sample, namely the $0811+5007,1401+5208$, and 2107-0620, also show flux variability and are listed in Table 3 . A wide range of viewing angles is estimated in their case. We have already mentioned that the lack of X-ray detection of 2107-0620 can be caused by the presence of the shielding gas (Wang et al. 2008). There are no X-ray observations of $1401+5007$, but the $0811+5007$ has been detected in X-rays showing no clear evidence for X-ray absorption from neutral hydrogen. As an explanation, Wang et al. (2008) suggested that if absorption is present in this source it must be more complex or that the X-rays come from the radio jet outside the shielding gas. Interestingly, the BAL disappearance has recently been reported in $0811+5007$ (Filiz et al. 2012). Such variations of the BAL troughs could be caused by the disk-wind rotation or variations of shielding gas that lead to variations of ionizing-continuum radiation. However, the second explanation cannot be ruled out. As has already been discussed in the case of BALQSO 1045+352 (Kunert-Bajraszewska et al. 2009), the jet synchrotron self-Compton (SSC) emission can be quite significant in some quasars and may dominate the X-ray energy range, while the $\mathrm{X}$-ray emission from the corona is absorbed in a large part (see also Miller et al. 2009). The comprehensive study of $1045+352$ also revealed the re-start of the jet activity in this source with the viewing angle $\sim 30^{\circ}$ (Kunert-Bajraszewska et al. 2010a).

As expected from previous studies (Shankar et al. 2008; Ghosh \& Punsly 2007) the number of candidates for variable BALQSOs is small, but even this small number can be overestimated (Ofek \& Frail 2011). Although the methodology proposed by Zhou et al. (2006) is a good start in looking for variability of 
radio sources, only a long-term monitoring programme of flux density of candidate sources from Table 3 will be able to provide more accurate measurements of the variability time scale, value of the flux change, etc., and thereby the $\theta_{\max }$ value. Further observational evidence for the existence of boosted radio emission in some BALQSOs has been found (Reynolds et al. 2013; Berrington et al. 2013).

\subsection{Discussion}

Gregg et al. (2006) has noted the scarcity of BALs among the large-scale, lobe-dominated radio-loud quasars and a drop in the BI index with increasing radio-loudness of the sources. Based on these results they proposed an evolutionary scenario in which BALs are associated with an early stage of the quasar evolution when a young quasar emerges from the surrounding dust. The anticorrelation between BI index and radio-loudness has not been confirmed by the statistical studies of Shankar et al. (2008) based on the Trump et al. (2006) catalogue. However, as has been pointed out by Gibson et al. (2009) there is a paucity of quasars that are simultaneously strongly radio-loud and heavily absorbed. The bottom panel of Fig. 2 shows the distribution of balnicity index versus the radio-loudness parameter $\log R_{\mathrm{I}}$ for quasars selected by us from Gibson et al. (2009). It can be seen that the largest values of BI index occurs among objects with $\log R_{\mathrm{I}}<1.5$. The radio variability study of a sample of BALQSOs indicates that the same subgroup of BALQSOs has the widest range of viewing angles $\theta$ (Fig. 3). This range changes as a function of the radio-loudness parameter for the whole sample, which seems to be in agreement with recent results of the radio-loudness parameter as an orientation indicator (Kimball et al. 2011b). It is commonly believed that the division line on quasars and radio galaxies should be at $\theta=40-45^{\circ}$ (Barthel 1989; Ghisellini at al. 1993) and our limits $\left(<59-68^{\circ}\right)$ are consistent with that. Earlier Monte Carlo simulations of viewing angles of BALQSOs showed generally smaller values for both BAL and non-BALQSOs $\left(<33^{\circ}\right)$, but pointed out that the viewing angles of BALQSOs may extend $\sim 10^{\circ}$ farther from the radio jet axis than non-BAL objects (DiPompeo et al. 2012). Recent numerical simulations assuming a simple kinematic disk wind model can predict BAL spectra for a variety of viewing angles, as large as $\sim 80^{\circ}$ (Higginbottom et al. 2013).

We would like, however, to draw attention to the fact that the most numerous $\log R_{\mathrm{I}}<1.5$ subgroup of radio-loud BALQSOs is not exclusively connected with strong absorption. In practice, we observe a very wide range of BI indices among them and we conclude that orientation is only one of the factors influencing the measured absorption. In other words, the largest viewing angles that still guarantee the source to be classified as a quasar do not guarantee the largest absorption in every case. Spectroscopic and variability studies of BAL features shows that complex behaviour of wind outflows, from minor depth changes within the BAL profile to BAL disappearance (Capellupo et al. 2013; Filiz et al. 2012), is present in BALQSOs and can be responsible for a wide range of covering factors within objects from the same group characterized by e.g. the radio-loudness parameter $R_{\mathrm{I}}$.

The selection criteria of our sample $\left(S_{\text {FIRST }}>2 \mathrm{mJy}\right)$, based on the Gibson et al. (2009) catalogue, led to only $\sim 15 \%$ of the sources being formally radio-quiet $(\log R<1)$. In fact, this number is much larger (Welling et al. 2014), although deep radio observations and population analysis show that all quasars have some minimum level of radio flux (White et al. 2007; Kimball et al. 2011a; Condon et al. 2013). The origin of the

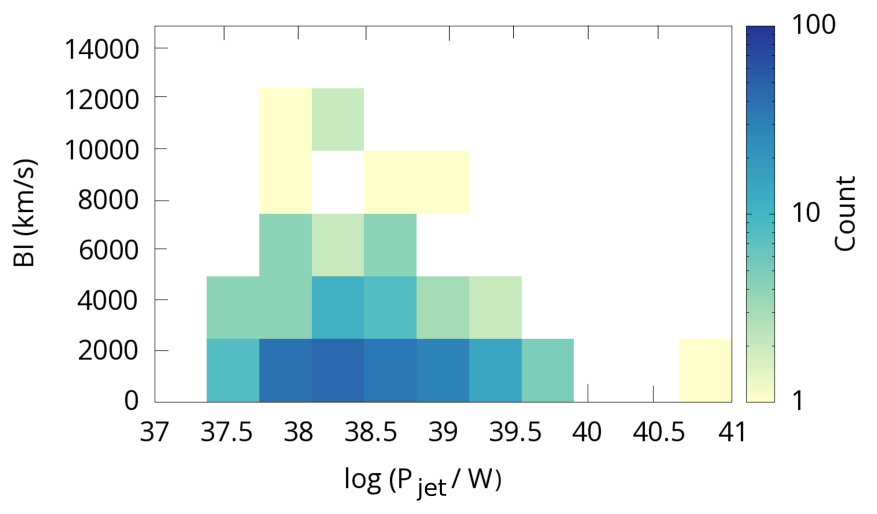

Fig. 4. Jet power vs. the value of modified balnicity index $\mathrm{BI}_{0}\left(\mathrm{C}_{\mathrm{IV}}\right)$ for the whole sample of radio-loud BALQSOs selected from Gibson et al. (2009) as described in Sect. 2. The jet power is calculated for $f=10$ (Sikora et al. 2013).

radio emission in sources weaker than $2.4 \mathrm{mJy}$ could be stellar (Condon et al. 2013), while in the case of stronger objects - with $\log L_{1.4 \mathrm{GHz}}>23.4 \mathrm{~W} \mathrm{~Hz}^{-1}$ - the radio activity is powered not by starbursts (Kimball et al. 2011a; Ulvestad et al. 2005) but by AGNs or may originate in shocks formed by the collision of the disk wind and the interstellar medium (Sikora 2009; Zakamska \& Greene 2014). The radio properties of the luminous BALQSOs presented in this paper indicate that their radio emission is of AGN origin rather than stellar. However, when considering our whole sample it can be seen that many of the radio-loud BALQSOs are intermediate-radio objects with $1.4 \mathrm{GHz}$ luminosities in the FR I-FR II transition region $(24<$ $\left.\log L\left[\mathrm{~W} \mathrm{~Hz}^{-1}\right]<25.5\right)$. Some of them, with flat spectrum, could in fact be boosted weak sources (Falcke et al. 1996; Berrington et al. 2013), but the majority have steeper spectra than nonBALQSOs (DiPompeo et al. 2011; Bruni et al. 2012). What is more, they are probably young objects at the beginning of their evolution.

Studies of the compact radio sources suggest that their evolution is determined by the properties of their central engine: strength, accretion mode, excitation level of the ionized gas, and the ISM (Kunert-Bajraszewska et al. 2010b; Kunert-Bajraszewska \& Labiano 2010). Low luminosity young AGNs may develop diffuse, large-scale structures (Gawroński et al. 2006; Cegłowski et al. 2013) as their weak jets are disrupted before escaping their host galaxies. They may undergo disrupted evolution many times, before finally they will be able to escape the host galaxy and evolve further. Such behaviour explains the excess of compact AGNs compared to large-scale FR Is and FR IIs (O’Dea 1998; Kunert-Bajraszewska et al. 2010b). According to Shankar et al. (2008) there is no difference between the fraction of large-scale sources among the BALQSOs and among the overall sample of radio objects at fixed radio power. Therefore, we suggest that the short lifetime of some compact AGNs could also explain the low number of extended "adult" radio sources among the BALQSOs. In this sense some of the compact AGNs (and hence BALQSOs) can be also considered as the re-activated objects as proposed by a few authors (Kunert-Bajraszewska et al. 2010a; Bruni et al. 2013; Hayashi et al. 2013) based on the larger-scale extended emission found around the new compact source.

According to the analysis performed by (Willott et al. 1999), the radio luminosity of a large-scale radio source is approximately proportional to the jet power. Adopting the modifications 
made by Sikora et al. (2013), we use their equation $P_{\text {jet }} \sim$ $10^{2} v_{1.4 \mathrm{GHz}} L_{1.4 \mathrm{GHz}}(f / 3)^{3 / 2}-$ where factor $f$ is in the range $1-20$ - to calculate the jet power of the selected BALQSOs. We then plot the $1.4 \mathrm{GHz}$ jet power versus the value of modified balnicity index $\mathrm{BI}_{0}\left(\mathrm{C}_{\mathrm{IV}}\right)$ and present it in Fig. 4. There is no formal correlation between these two parameters, but the trend of the highest values of BI index to be associated with the lower jet powers can be seen in accordance with the scenario discussed above ${ }^{2}$.

\section{Summary}

We have presented EVN and VLBA observations of a sample of radio-loud BALQSOs together with the variability studies of some of them. We find the following:

- The peak of the radio-loundness parameter $\left(\log R_{\mathrm{I}}\right)$ distribution of our sample of compact BALQSOs is in the range $1-1.5$. The number of sources decreases with the increasing radio flux density and $\log R_{\mathrm{I}}$. Therefore, the BALQSOs that we are able to image with the VLBI are the most luminous ones, which constitute the minority of the radio-loud BALQSO population.

- Nine of the ten sources from the VLBI sample are strong radio-loud BALQSOs with $1.4 \mathrm{GHz}$ flux densities in the range $20-80 \mathrm{mJy}$ and $\log R_{\mathrm{I}}>1.8$. The one exception is the low redshift source 2107-0620. All sources have very compact sizes $(<50 \mathrm{pc})$, but most of them have been resolved at $5 \mathrm{GHz}$ showing one-sided, probably core-jet structures, typical of quasars.

- Distribution of the BI index versus the radio-loudness parameter, $\log R_{\mathrm{I}}$, shows that the strongest absorption is associated with the lower values of the radio-loudness parameter, $\log R_{\mathrm{I}}<1.5$. The $R_{\mathrm{I}}$ parameter is considered an indicator of orientation and if this is the case the values of $\log R_{\mathrm{I}}<1.5$ can mean large viewing angles. However, the large span of BI values in the each bin of the radio-loudness parameter indicates that orientation is only one of the factors that influence the measured absorption.

- Many of the radio-loud BALQSOs are intermediate- or low-power radio objects with $1.4 \mathrm{GHz}$ luminosities in the FR I-FR II transition region and below it. They are powered by the AGN and their compact sizes may imply young age. Studies of the low luminosity compact radio sources indicate that many of them can be short-lived objects that undergo the GPS/CSS phase of activity many times before they become large-scale FR I or FR II sources. Such intermittent behaviour may also account for the scarcity of extended radio sources among the BALQSOs.

- The fact that the broad absorption lines are present mostly in the low-power radio objects may suggest an anticorrelation between the power of the jet and the value of the absorption and an indirect correlation with the process responsible for BAL phenomenon. We do not find such a relationship in our data; however, there is a hint in our analysis that the highest values of $\mathrm{BI}$ index are associated with the lower jet powers in BALQSOs.

\footnotetext{
2 The Willott formula is only accurate if there are relaxed radio lobes. Since most of our sources have sub-galactic sizes, the possible jet-dense medium interaction can increase the radio luminosity and thus the calculated jet powers can be overestimated. This, however, should affect the whole group equally and will not change the visible trend.
}

Acknowledgements. This work was supported by the National Scientific Centre under grant DEC-2011/01/D/ST9/00378. The research leading to these results has received funding from the European Commission Seventh Framework Programme (FP/2007-2013) under grant agreement No 283393 (RadioNet3). The European VLBI Network is a joint facility of European, Chinese, South African, and other radio astronomy institutes funded by their national research councils. The National Radio Astronomy Observatory is a facility of the National Science Foundation operated under cooperative agreement by Associated Universities, Inc. The research described in this paper makes use of Filtergraph, an online data visualization tool developed at Vanderbilt University through the Vanderbilt Initiative in Data-intensive Astrophysics (VIDA).

\section{Appendix A: Brightness temperature and viewing angle}

\section{A.1. Brightness temperature}

The observed flux density of a spherical or cylindrical source with radius $r$ is proportional to the source surface and inverse proportional to the square of the luminosity distance $\left(D_{\mathrm{L}}\right)$

$F_{v}=\frac{\pi r^{2}}{D_{\mathrm{L}}^{2}} I_{v}$,

where $I_{v}$ is the intensity of the emission. For a source that moves with relativistic velocity $(v \lesssim c)$ at extragalactic distance, we have to transform this parameter from the source comoving frame (primed quantities) to the observer's frame $I_{v}=$ $(1+z) \delta^{3} I_{v^{\prime}}^{\prime}$, where $\delta$ is the Doppler factor and the frequency transformation has the form

$v^{\prime}=\frac{(1+z)}{\delta} v$.

In the Rayleigh-Jeans limit $(h v \ll k T)$ the intensity of the synchrotron emission can be approximated by a simple relation $I_{\gamma^{\prime}}^{\prime}=2 k_{\mathrm{B}} v^{\prime} T_{\mathrm{b}} / c^{2}$, where $T_{\mathrm{b}}$ is the brightness temperature, which at radio frequencies should be comparable with the electron temperature $T_{\mathrm{e}}$, where the electron energy is given by $E=\gamma m_{\mathrm{e}} c^{2}=3 k_{\mathrm{B}} T_{\mathrm{e}}$. Substituting the above relations in the initial equation we obtain

$F_{v}=\frac{2 k_{\mathrm{B}}}{c^{2}} \frac{\pi r^{2}}{D_{\mathrm{L}}^{2}}(1+z)^{3} \delta v^{2} T_{\mathrm{b}}$.

It is useful to use the observed angular size of a source $(\theta)$ instead of the luminosity distance $D_{\mathrm{L}}=(1+z)^{2} D_{\mathrm{A}}$, where $D_{\mathrm{A}}$ is the angular distance. The angular distance can be approximated by $D_{\mathrm{A}} \simeq 2 r / \theta$, which finally gives

$F_{v}=\frac{k_{\mathrm{B}} \pi}{2 c^{2}} \frac{\delta}{1+z} \theta^{2} v^{2} T_{\mathrm{b}}$.

If the observed flux density $\left(F_{\gamma}\right)$ exceeds value of the flux density $\left(F_{v}^{12}\right)$ obtained for the maximum possible temperature $\left(T_{\mathrm{b}} \simeq\right.$ $\left.10^{12} \mathrm{~K}\right)$, then we can estimate a minimum value of the Doppler factor

$\delta \geqslant \frac{F_{v}}{F_{v}^{12}}$,

assuming that the observed excess comes from the beaming effect.

\section{A.2. Variability time scale}

An upper limit for the source size may be independently estimated from observed variability time scales. Time of a flux density variation $\left(\Delta t^{\prime}\right)$ in the comoving frame cannot be faster 


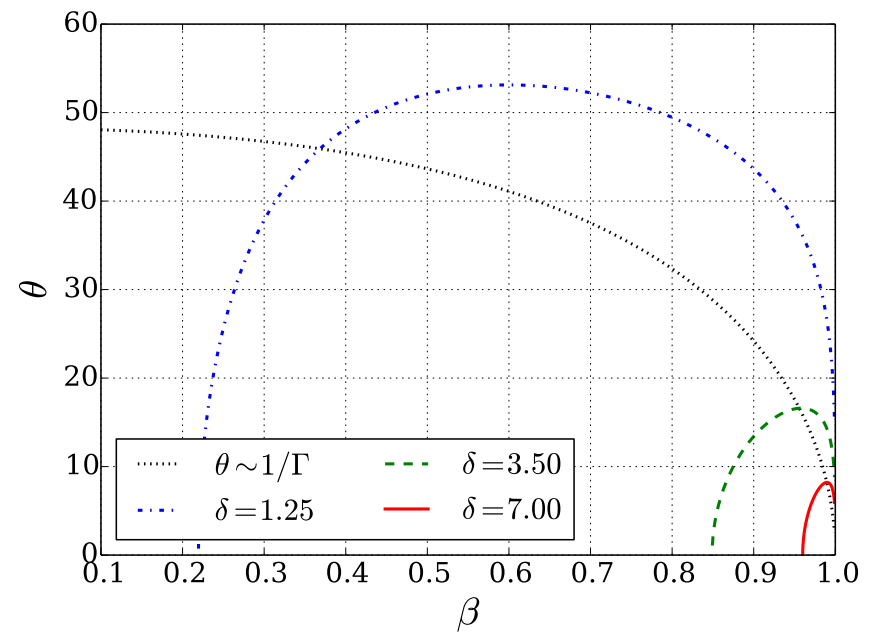

Fig. A.1. Viewing angle $\theta$ versus the jet velocity $\beta$ calculated from Eq. (A.8).

than the source crossing time $(r / c)$. Therefore, using the time transformation $\left(t=(1+z) \Delta t^{\prime} / \delta\right)$ we can write

$r \leqslant \frac{\delta}{1+z} c \Delta t$

where $\Delta t$ is the variability time scale in the observer's frame. Adopting the previously derived Eq. (A.3) we can write another constraint for the observed flux,

$F_{v} \leqslant 2 k_{\mathrm{B}} \pi \frac{\delta^{3}}{(1+z)^{3}} \frac{1}{D_{\mathrm{A}}} \Delta t^{2} v^{2} T_{\mathrm{b}}$.

If the observed flux exceeds the value calculated from this relation for $T_{\mathrm{b}}=10^{12} \mathrm{~K}$ or $T_{\mathrm{b}}=10^{11} \mathrm{~K}$ (Lähteenmäki et al. 1999), then very likely the emission is beamed and again we can obtain a lower limit for the Doppler factor.

\section{A.3. Viewing angle}

The Doppler factor depends on the source velocity $(\beta=v / c)$ and the viewing angle $(\theta)$. Therefore, an independent estimation of this parameter does not provide a direct value of the viewing angle. For a given value of the Doppler factor we can derive only an upper limit for the viewing angle

$\cos \theta \leqslant \max \left[\frac{\delta-\sqrt{1-\beta^{2}}}{\delta \beta}\right]$.

For example, the Doppler factor value $\delta \geqslant 2$ limits $\theta$ to the $0-30^{\circ}$ range. We note that the viewing angle cannot be larger than the half of the emission beam angle $\phi \sim 1 / \Gamma=\sqrt{1-\beta^{2}}$ [rad]. However, this condition is valid only for $\delta \gtrsim 2$ and does not give additional constraints to the $\theta$.

\section{References}

Barthel, P. D. 1989, ApJ, 336, 606

Becker, R. H., White, R. L., Gregg, M. D., et al. 2000, ApJ, 538, 72

Berrington C. R., Brotherton, M. S., Gallagher, S. C., et al. 2013, MNRAS, 436, 3321

Bruni, G., Mack, K.-H., Salerno, E., et al. 2012, A\&A, 542, A13

Bruni, G., Dallacasa, D., Mack, K.-H., et al. 2013, A\&A, 554, A94
Capellupo, D. M., Haman, F., Shields, J. C., Halpern, J. P., \& Barlow, T. A. 2013, MNRAS, 429, 1872

Cegłowski, M., Gawroński, M., \& Kunert-Bajraszewska, M. 2013, A\&A, 557, A75

Cegłowski, M., Kunert-Bajraszewska, M., \& Roskowiński, 2015, MNRAS, 450, 1123

Condon, J. J. 1992, ARA\&A, 30, 575

Condon, J. J., Cotton, W. D., Greisen, E. W., et al. 1998, AJ, 115, 1693

Condon, J. J., Kellermann, K. I., Kimball A. E., Ivezić, Ž., \& Perley, R. A. 2013, ApJ, 768, 37

Deller, A., Brisken, W. F., Phillips, C. J., et al. 2011, PASP, 123, 275

DiPompeo, M. A., Brotherton, M. S., De Breuck, C., et al. 2011, ApJ, 743, 71

DiPompeo, M. A., Brotherton, M. S., De Breuck, C., et al. 2012, ApJ, 752, 6

Doi, A., Kawaguchi, Noriyuki, Kono, Yusuke, et al. 2009, PASJ, 61, 1389

Elvis, M. 2000, ApJ, 545, 63

Falcke, H., Sherwood, W., \& Patnaik, A. R. 1996, ApJ, 471, 106

Fanti, C., Fanti, R., Dallacasa, D., et al. 1995, A\&A, 302, 317

Filiz, Ak, N., Brandt, W. N., Hall, P. B., et al. 2012, ApJ, 757, 114

Fine, S., Jarvis, M. J., \& Mauch, T. 2010, MNRAS, 412, 213

Ganguly, R., Brotherton, M. S., Cales, S., et al. 2007, ApJ, 665, 990

Gawroński, M., \& Kunert-Bajraszewska, M. 2011, PoS(X EVN Symp.)037

Gawroński, M., Marecki, A., Kunert-Bajraszewska, M., \& Kus, A. J. 2006, A\&A, 447, 63

Gibson, R. R., Jiang, Linhua, Brandt, W. N., et al. 2009, ApJ, 692, 758

Ghisellini, G., Padovani, P., Celotti, A., \& Maraschi, L. 1993, ApJ, 407, 65

Ghosh, K. K., \& Punsly, B. 2007, 661, L139

Gregg, M. D., Becker, R. H., \& de Vries, W. 2006, ApJ, 641, 210

Hayashi, T., Doi, A., \& Nagai, H. 2013, ApJ, 772, 4

Hewett, P. C., Foltz, \& C. B. 2003, AJ, 125, 1784

Higginbottom, N., Knigge, C., Long, K. S., Sim, S. A., \& Matthews, J. H. 2013, MNRAS, 436, 1390

Jiang, D. R., \& Wang, T. G. 2003, A\&A, 397, L13

Knigge, C., Scaringi, S., Goad, M. R., \& Cottis, C. E. 2008, MNRAS, 386, 1426

Kimball A. E., Kellermann, K. I., Condon, J. J., Ivezić, Ž., \& Perley, R. A. 2011a, ApJ, 739, L29

Kimball A. E., Ivezić, Ž., Wiita, P. J., \& Schneider, D. P. 2011b, AJ, 141, 182

Kunert-Bajraszewska, M., \& Labiano, A. 2010, MNRAS, 408, 2279

Kunert-Bajraszewska, M., \& Marecki, A. 2007, A\&A, 469, 437

Kunert-Bajraszewska, M., Siemiginowska, A., Katarzyńki, K., Janiuk, A. 2009, ApJ, 705, 1356

Kunert-Bajraszewska, M., Janiuk, Agnieszka, Gawroński, M. P., \& Siemiginowska, A. 2010a, ApJ, 718, 1345

Kunert-Bajraszewska, M., Gawro'nski, M. P., Labiano, A., \& Siemiginowska, A. 2010b, MNRAS, 408, 2261

Lähteenmäki, A, Valtaoja, E., \& Wiik, K. 1999, ApJ, 511, 112

Liu, Y., Jiang, D. R., Wang, T. G., \& Xie, F. G. 2008, MNRAS, 391, 246

Miller, B. P., Brandt, W. N., Gibson, R. R., Garmire, G. P., \& Shemmer, O. 2009, ApJ, 702, 911

Montenegro-Montes, F. M., Mack, K.-H., Benn, C., et al. 2008, PoS(IX EVN Symp.)019

Murray, N., Chiang, J., Grossman, S. A., \& Voit, G. M. 1995, ApJ, 451, 498

O'Dea, C. P. 1998, PASP, 110, 493

Ofek, E. O., \& Frail, D. A. 2011, ApJ, 737, 45

Schneider, D. P., Hall, P. B., Richards, G. T., et al. 2007, AJ, 134, 102

Shankar, F., Dai, X., \& Sivakoff, G. R. 2008, ApJ, 687, 859

Shepherd, M. C. 1997, in Astronomical Data Analysis Software and Systems VI, eds. G. Hunt, \& H. Payne (San Francisco, CA: ASP), ASP Conf. Ser. 125, 77

Sikora, M. 2009, AN, 330, 291

Sikora, M., Kozieł-Wierzbowska, D., Madejski, G. M., \& Asari, N. V. 2013, ApJ, 765,62

Readhead, A. C. S. 1994, ApJ, 426, 51

Reynolds, C., Punsly, B., \& O'Dea, C. P. 2013, 773, L10

Trump, J. R., Hall, P. B., Reichard, T. A., et al. 2006, ApJS, 165, 1

Ulvestad, J. S., Antonucci, R. R. J., \& Barvainis, R. 2005, ApJ, 621, 123

Wang, J., Jiang, P., Zhou, H., et al. 2008, ApJ, 676, L97

Welling, C. A., Miller, B. P., Brandt, W. N., Capellupo, D. M., \& Gibson, R. R. 2014, MNRAS, 440, 2474

Weymann, R. J., Morris, S. L., Foltz, C. B., \& Hewett, P. C. 1991, ApJ, 373, 23

White, R. L., Becker, R. H., Helfand, D. J., \& Gregg, M. D. 1997, ApJ, 475, 479

White, R. L., Helfand, D. J., Becker, R. H., Glikman, E., \& de Vries, W. 2007, ApJ, 654, 99

Willott, C. J., Rawlings, S., Blundell, K. M., \& Lacy, M. 1999, MNRAS, 309, 1017

Wills, B. J., \& Brotherton, M. S. 1995, ApJ, 448, L81

Zakamska, N. L., \& Greene, J. E. 2014, MNRAS, 442, 784

Zhou, H., Wang, T., Wang, H., et al. 2006, ApJ, 639, 716 